\title{
Enhancement of Multilayer Perceptron Model Training Accuracy through the Optimization of Hyperparameters: A Case Study of the Quality Prediction of Injection Molded Parts
}

\section{Kun-Cheng Ke}

National Kaohsiung University of Science and Technology

Ming-Shyan Huang ( $\nabla$ mshuang@nkust.edu.tw)

National Kaohsiung University of Science and Technology https://orcid.org/0000-0002-0477-7357

\section{Research Article}

Keywords: Activation function, hyperparameter, injection molding, learning rate, machine learning, momentum, quality prediction Sigmoid function, Stochastic Gradient Descent

Posted Date: July 13th, 2021

DOl: https://doi.org/10.21203/rs.3.rs-685234/v1

License: (c) (i) This work is licensed under a Creative Commons Attribution 4.0 International License. Read Full License

Version of Record: A version of this preprint was published at The International Journal of Advanced Manufacturing Technology on September 26th, 2021. See the published version at https://doi.org/10.1007/s00170-021-08109-9. 


\title{
Enhancement of multilayer perceptron model training accuracy
}

\section{through the optimization of hyperparameters: A case study of the quality prediction of injection molded parts}

\author{
Kun-Cheng Ke \\ Department of Mechatronics Engineering, National Kaohsiung University of Science and \\ Technology, 1 University Road, Yanchao Dist., Kaohsiung City 824, Taiwan \\ Email:kcke@nkust.edu.tw \\ Ming-Shyan Huang* \\ Department of Mechatronics Engineering, National Kaohsiung University of Science and \\ Technology, 1 University Road, Yanchao Dist., Kaohsiung City 824, Taiwan \\ Email:mshuang@nkust.edu.tw \\ *Corresponding to: M.-S. Huang (Email: mshuang@nkust.edu.tw; Tel: +886-6011000 ext. \\ 32219; Fax: +886-7-6011066)
}




\begin{abstract}
Injection molding has been broadly used in the mass production of plastic parts and must meet the requirements of efficiency and quality consistency. Machine learning can effectively predict the quality of injection molded part. However, the performance of machine learning models largely depends on the accuracy of the training. Hyperparameters such as activation functions, momentum, and learning rate are crucial to the accuracy and efficiency of model training. This research further analyzed the influence of hyperparameters on testing accuracy, explored the corresponding optimal learning rate, and provided the optimal training model for predicting the quality of injection molded parts. In this study, stochastic gradient descent (SGD) and stochastic gradient descent with momentum were used to optimize the artificial neural network model. Through optimization of these training model hyperparameters, the width testing accuracy of the injection product improved. The experimental results indicated that in the absence of momentum effects, all five activation functions can achieve more than $90 \%$ of the training accuracy with a learning rate of 0.1 . Moreover, when optimized with the SGD, the learning rate of the Sigmoid activation function was 0.1, and the testing accuracy reached 95.8\%. Although momentum had the least influence on accuracy, it affected the convergence speed of the Sigmoid function, which reduced the number of required learning iterations $(82.4 \%$ reduction rate). Optimizing hyperparameter settings can improve the accuracy of model testing and markedly reduce training time.
\end{abstract}

Keywords: Activation function, hyperparameter, injection molding, learning rate, machine learning, momentum, quality prediction Sigmoid function, Stochastic Gradient Descent. 


\section{Introduction}

Injection molding is a key step in polymer processing and comprises the five stages of clamping, filling, packing, cooling and plasticizing, and demolding. When polymer materials are molded, the melt and mold temperatures, filling speed, packing pressure, and time are the primary factors that affect the quality of the parts [1]. In particular, polymers are temperature sensitive; different temperatures exhibit distinct rheological properties that affect the flow properties of the melt. Melt filling is driven by pressure, and the required pressure is related to the setting of the forward screw speed. A filling speed that is too low or high results in short shots and jetting problems, respectively. In addition, the holding pressure (also called postfilling) can compensate for the gap between the polymer melt after cooling and shrinking in the mold cavity, ensuring that the finished product meets the size requirements. Therefore, machine settings also influence the quality of the final product. However, under the same machine settings, because of the adverse effects of actual machine movement, material stability, and environmental factors, this quality cannot be guaranteed.

To determine the actual flow behavior of the polymer melt, pressure sensors installed on the surface of the mold cavity can measure the pressure changes of the melt during molding [24]. The temperature distribution of the melt on the mold surface directly affects the quality of the product. The use of a composite sensor to track melt pressure and temperature changes during the injection molding process reveals the relationship between pressure and temperature, which can be monitored to further control the volume change in injection molded products [4]. Furthermore, some researchers have employed nondestructive ultrasonic sensing technology to measure changing melt pressure in the mold during the injection process, through which the melt pressure state can be monitored without damaging the mold structure and the various stages of the molding process can be identified [5].

Following the capturing of the molding data, diverse methods can be applied to predict quality, including domain knowledge, statistical methods, and artificial intelligence techniques. 
In regards to domain knowledge, the pvT theorem is crucial to describing the specific volume state of the polymer melt corresponding to pressure and temperature changes during the injection molding process. The optimum setting of the pvT molding path (e.g., the use of scientific molding methods [6-9]) assists in obtaining optimal product quality.

Statistics-based mathematical models can describe the relationship between process parameters and part quality. Among them, the Taguchi experimental method combined with analysis of variance or correlation coefficient is widely used to identify the ideal combination of injection molding parameters. This method can be used to reduce the number of experiments and determine the factors that reduce manufacturing costs and achieve stable quality goals [1014]. The emergence of artificial intelligence has also offered highly nonlinear fitting possibilities. Through the establishment and testing of different training models, it can be effectively applied to various scenarios. Artificial neural networks (ANNs) enable users to rapidly create artificial networks and quality prediction solutions through appropriate hyperparameter adjustments. These adjustments link a large amount of process parameter information with resultant molding quality [15-19]. ANN technology can be employed in the learning process of different data types from large volumes of data to achieve clustering, classification, prediction, and regression functions. In particular, ANNs are suitable for modeling tasks involving nonlinear relationships, such as thermoplastic injection molding processes with complex viscoelastic material behavior and nonlinear relationships between quality, process, and machine parameters. Hyperparameters, including the hidden layer architecture, activation functions, optimization solver, learning rate, and momentum, play key roles in model learning. Common optimization solvers include the stochastic gradient descent (SGD) $[20,21]$, stochastic gradient descent with momentum (SGDM) $[22,23]$ and Adam [24, 25]. Learning rate and momentum are both influential in the quality and speed of model learning but are rarely studied in the literature.

To explore the influence of hyperparameters in the actual training process on model training 
speed, this research examined the injection molding of integrated circuit (IC) trays, and extracted the pressure curve indicating the quality of the part into the quality index. Through application of the index and width of the part to the input and output layers of the neural model, respectively, the two optimization solvers SGD and SGDM were explored. Among them, the activation function, learning rate, and momentum varied, and their influence on the accuracy, convergence speed, and oscillation of the training model was analyzed to provide a reference for adjustment.

\section{Methods}

\subsection{Multilayer perceptron model}

The multilayer perceptron (MLP) model is a supervised ANN learning model with a forward propagation structure that maps a set of input vectors to a set of output vectors. An MLP can be regarded as a directed graph composed of multiple node layers, with each layer fully connected to the next layer. The MLP model contains three layers, namely the input, hidden, and output layers. Except for the input node, each node is a neuron with a summation function and activation function. The activation function, expressed in Eq. (1), is a nonlinear function used to map the summation function $(\boldsymbol{x} \boldsymbol{w}+b)$ to the output value $\boldsymbol{y}$. The terms $\boldsymbol{x}, \boldsymbol{w}, b$, and $\boldsymbol{y}$ represent the input vector, weighting vector, bias, and output value, respectively.

$$
\boldsymbol{y}=\varphi(\boldsymbol{x} \boldsymbol{w}+b)
$$

The weighting values range between o and 1 . These values change with the training data and represent the memory of the neural network related to the input and output model training.

\subsection{SGDM}

SGDM is an optimized solution algorithm typically employed in MLP model training. As described in Eqs. (2) and (3), the SGD optimization algorithm, also known as the steepest descent method, generates function solutions in reference to the opposite direction of the gradient and step distance (or learning rate, $\alpha$ ) to iteratively search the weighting value $(w)$. Although the weights can be updated iteratively using the SGD algorithm, if multiple local 
minimums are present in the function, the local minimum or saddle point can be searched, but the global minimum cannot be obtained. This consequently halts the training iterations and leads to erroneous learning results.

The SGDM algorithm that combines SGD and momentum $(\beta)$ adjustment has attracted considerable attention. The SGDM algorithm detailed in Eqs. (4) and (5) takes $\beta$ into account, and thus, the optimization algorithm can jump out of the local minimum during the model training process. This procedure enables the entire iteration to stably converge to the minimum value of the loss function.

$$
\begin{gathered}
\boldsymbol{w}_{t+1}=\boldsymbol{w}_{t}-\alpha \times \boldsymbol{g}_{t} \\
\boldsymbol{g}_{t}=-\frac{\partial \boldsymbol{L o s s}}{\partial \boldsymbol{w}_{\boldsymbol{t}}} \\
\boldsymbol{w}_{t+1}=\boldsymbol{w}_{t}-\alpha \times \boldsymbol{m}_{t} \\
\boldsymbol{m}_{\boldsymbol{t}}=\beta \times \boldsymbol{m}_{\boldsymbol{t}-\mathbf{1}}+(1-\beta) \times \boldsymbol{g}_{\boldsymbol{t}}
\end{gathered}
$$

\subsubsection{Learning rate}

The learning rate $(\alpha)$ determines the iteration speed of weight adjustment in the neural network according to the gradient loss function. That is, the smaller the learning rate is, the slower the decline along the loss gradient is. A lower learning rate can prevent potentially optimal values from being overlooked, thus obtaining higher training accuracy, but this process requires a longer convergence time. Generally, the setting of the learning rate depends on experience, model size, and numerical complexity. Even for the same training model, when faced with input values of diverse dimensions, the convergence of the optimal learning rate must also change. Therefore, the learning rate must be adjusted for various data conditions. Error! Reference source not found. depicts the optimization status for various learning rates. A low learning rate has a slow convergence speed but ensures that the minimum is identified at each step of training to obtain optimal training accuracy. By contrast, a high learning rate can accelerate the convergence speed but may fix on a suboptimal solution. 


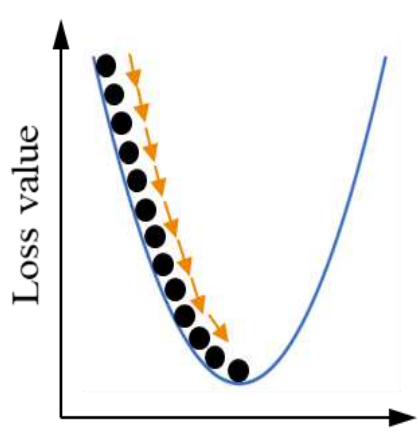

(a)

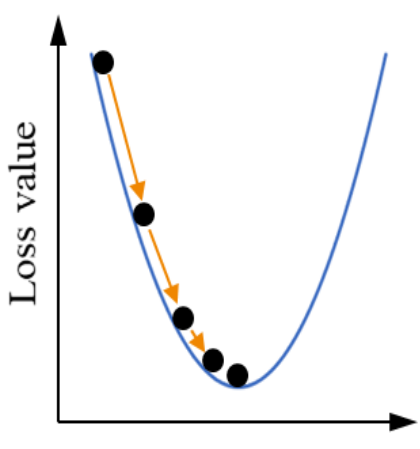

(b)

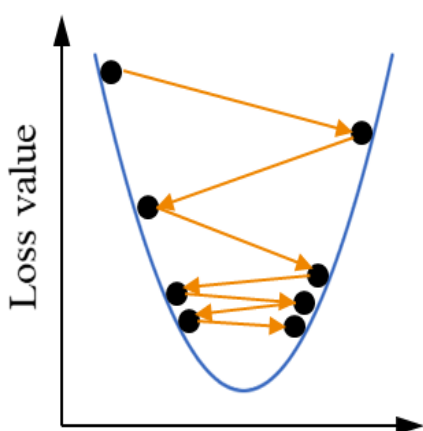

(c)

Fig. 1 Status of various learning rates: (a) too low; (b) appropriate; (c) too high

\subsubsection{Momentum}

The conventional SGD algorithm calculates the steepest descent gradient to rapidly search for the minimum value of the objective function. However, such a simplistic strategy cannot prevent the failure of local minimums or saddle points, which results in false stops during the iterative search process. The introduction of momentum $(\beta)$ in the SGD algorithm can overcome these shortcomings. Figure 2 illustrates the path of searching the minimum value, where the momentum represents the magnitude of the next movement, with the direction based on the current position.

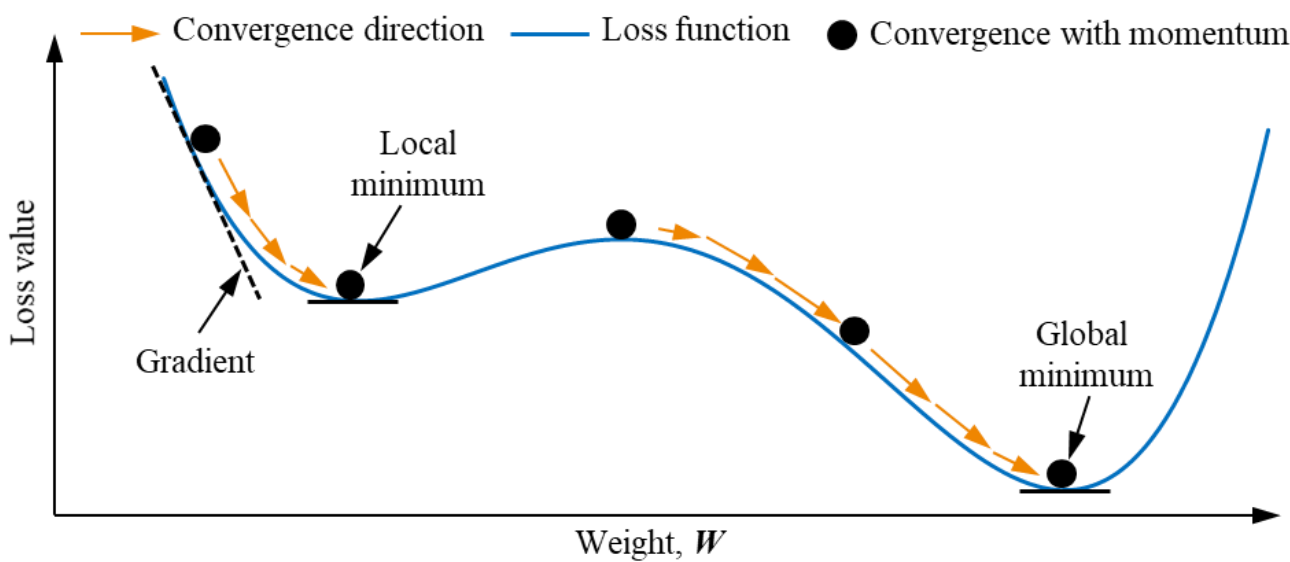

Fig. 2 Path of searching the minimum value

\subsubsection{Activation function}

The activation function, described in Eq. (1), in a neural network model transforms the 
inputs into outputs within a certain range and is added to the ANN to assist the network in learning complex patterns in the data. Specifically, these functions introduce nonlinear physical systems to ANNs. Without an activation function, a neural network acts as a linear regression with limited learning ability. To efficiently and accurately learn the nonlinear state of the quality of the injection molded parts in correspondence to the process parameters, a suitable activation function is necessary. The derivatives also play vital roles in the weight update. Various activation functions perform this task differently. Figure 3 presents the various activation functions used in the ANN model, and Eq. (6) describes a commonly used Sigmoid activation function that converts the real value into the range of 0 to 1 . When the input number is large, the result is close to 1 and, when entering a small negative number, the result is close to 0 . This function can accurately indicate whether the neuron is stimulated, with 0 and 1 referring to low and full activation, respectively. The Sigmoid function acts as the last layer of the machine learning model to convert the output into a probability score, which can be simpler to use and interpret. If the inputs are relatively large or small, the Sigmoid function induces a saturation effect in which the neuron resembles a dead state.

$$
f_{(x)}=\frac{1}{1+e^{-x}}
$$

The Tanh activation function detailed in Eq. (7) and Fig. 3(b) compresses the input value within a range of -1 to 1 . This function is similar to the Sigmoid function; thus, when the number of inputs is relatively large or small, the neuron resembles a dead state.

$$
f_{(x)}=\frac{e^{x}-e^{-x}}{e^{x}+e^{-x}}
$$

When using the Sigmoid or Tanh activation functions, the gradient decreases as the number of layers increases. This reduces the gradient value of the initial layer, and these layers thus cannot be correctly learned; when the depth of the network moves the value to 0 , their gradient tends to vanish, which is known as the vanishing gradient problem [26].

The rectified linear unit (ReLU) presented in Fig. 3(c) and Eq. (8) is characterized by the 
value of the first derivative located at 0 and 1. Compared with the Sigmoid and Tanh activation functions, the gradient of the ReLU is simple, does not face saturation, and converges more rapidly. However, when a large error gradient accumulates and leads to a large update of the neural network weights, an exploding gradient occurs, resulting in unstable learning. Moreover, a dying ReLU can become problematic when the gradient of all negative input values is 0 ; if a ReLU neuron is stuck on the negative side and consistently outputs 0 , it is considered "dead." Because the slope of the ReLU in the negative range is also 0, once the neuron becomes negative, it is unlikely to recover. Such neurons cannot play any role in distinguishing inputs and are essentially useless.

$$
f_{(x)}= \begin{cases}0, & x<0 \\ x, & x \geq 0\end{cases}
$$

The Leaky ReLU activation function, described in Eq. (9) and Fig. 3(d), represents an improved version of the ReLU. This function primarily prevents the disappearance of the gradient generated when $\mathrm{x}$ is less than 0 . When a neuron is in an inactive state, it allows a nonzero gradient, where $C_{l}$ is a small positive constant.

$$
f_{(x)}=\left\{\begin{array}{rr}
C_{1} x, & x<0 \\
x, & x \geq 0
\end{array}\right.
$$

The exponential linear unit (ELU) activation function presented in Eq. (10) and Fig. 3(e) contains an adjustable positive constant $C_{2}$ to avoid the possible saturation of the ELU.

$$
f_{(x)}=\left\{\begin{array}{cc}
x, & x<0 \\
C_{2}\left(e^{x}-1\right), & x \geq 0
\end{array}\right.
$$

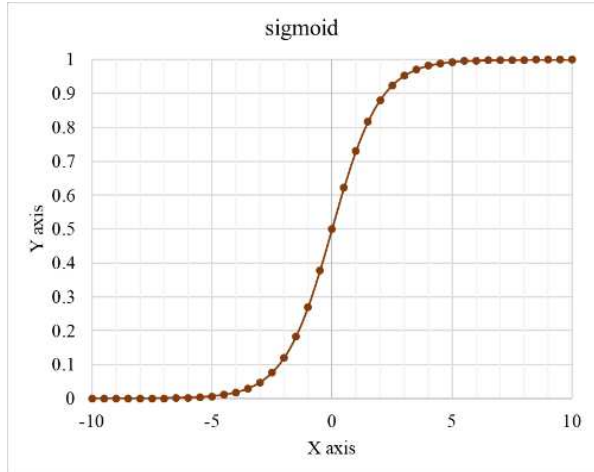

(a)

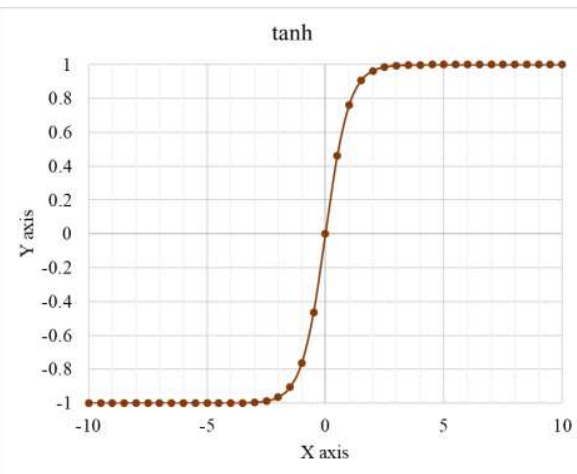

(b) 


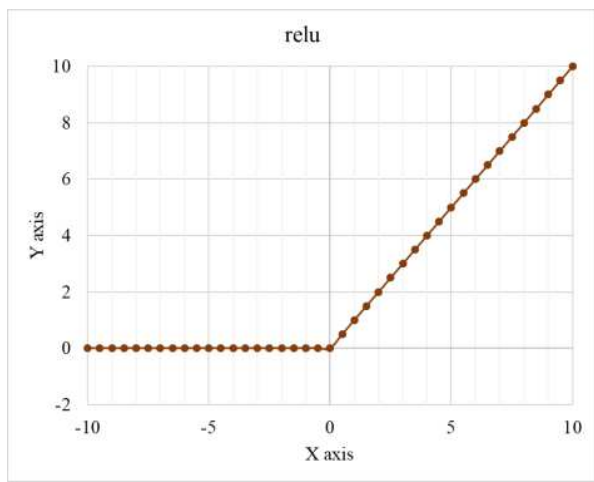

(c)

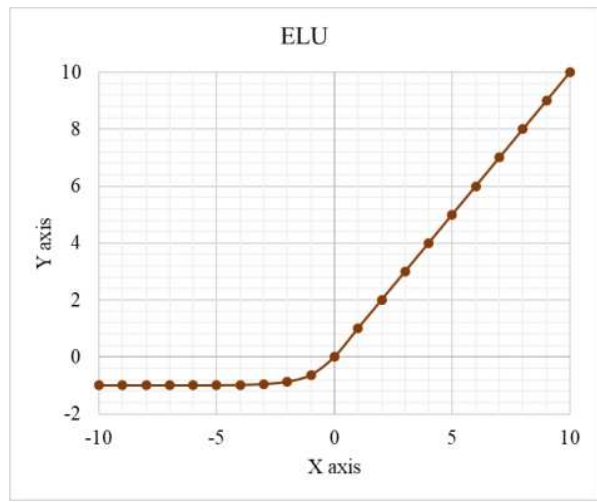

(e)

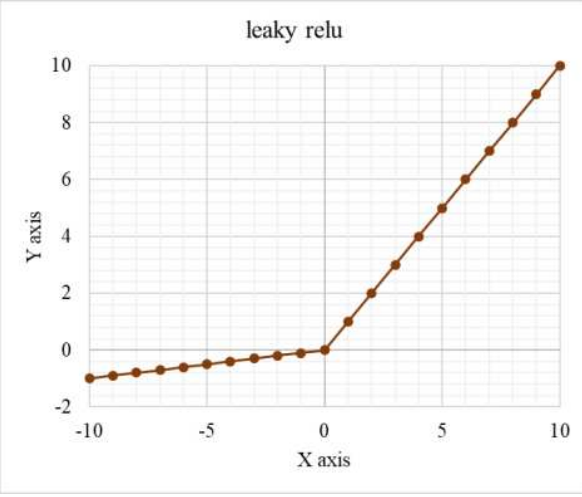

(d)

Fig. 3 Activation functions: (a) Sigmoid; (b) Tanh; (c) ReLu; (d) Leaky ReLU; (e) ELU

\subsection{Quality indices}

To predict the quality of parts in terms of molding condition changes and reduce the amount of calculation data in the experiment, this study combined domain knowledge and data mining technology to convert the injection molding data into quality indices. The correlation of these indices with the quality of the finished product was then calculated. Those that correlated highly were selected as input parameters to improve the prediction accuracy and effectively reduce the amount of calculation required during model training. The following four main quality indices were introduced in this study $[16,17]$ :

(1) First-stage holding pressure index $\left(P h_{\text {index }}\right)$ : This represents the average holding pressure in the first stage, as expressed in Eq. (11). The function of the holding pressure $(g)$ is to compensate for the volumetric shrinkage of the part caused by the cooling of the polymer melt. The holding pressure affects the geometric dimensions of injection molded parts and 
is crucial to precision injection molding.

$$
P h_{\text {index }}=\frac{1}{t_{1}-t_{0}} \int_{t_{0}}^{t_{1}} g d t
$$

(2) Peak pressure index $\left(P p_{\text {index }}\right)$ : This represents the maximum pressure during filling and compression, as described in Eq. (12). Injection molding constitutes a series of pressuredriven $(f)$ melt flow processes. The pressure not only determines the speed of the melt flow and its flow inertia but also affects the quality of the melt flowing into the mold cavity. Therefore, the maximum pressure index affects the quality and geometry of injection molded parts.

$$
P p_{\text {index }}=\operatorname{Max}(f)
$$

(3) Residual pressure drop index $\left(P r_{\text {index }}\right)$ : This indicates the average residual pressure drop in the cavity during cooling, as detailed in Eq. (13). The average residual pressure drop is related to the residual stress of the polymer. A residual pressure that is excessively high or low may cause geometric deformation or size shrinkage of the injection molded parts, respectively.

$$
\operatorname{Pr}_{\text {index }}=\frac{1}{t_{3}-t_{2}} \int_{t_{2}}^{t_{3}} f d t
$$

(4) Pressure integral index $\left(P I_{\text {index }}\right)$ : As expressed in Eq. (14), this indicates the integral value of the pressure curve with respect to time during the molding cycle, including the pressure changes during the melt filling process. This index is related to the total pressure characteristics of the polymer melt during the molding process. Variation in the index can reflect changes in part quality, particularly weight-related changes.

$$
P I_{\text {index }}=\int_{0}^{t_{3}} f d t
$$

These four indices have different ranges of values and are normalized between 0 and 1 . Using these indices as inputs for model training can support rapid convergence and high accuracy. 


\section{Experimental setup}

\subsection{Mold, machine, materials, and measurements}

In this experiment, an IC tray of $76 \times 76 \times 4.4 \mathrm{~mm}^{3}$ and flow length-to-thickness ratio of 124 was fabricated as the research carrier for method verification (Fig. 4). The experimental operation was conducted on an all-electric injection machine (CT100; Fu-Chun Shin, Tainan, Taiwan) with a maximum clamping force of 100 tons The processed material was acrylonitrile butadiene styrene (PA-756; Chi-Mei, Tainan, Taiwan). To collect the physical information on the polymer melt flow behavior within the mold cavity, two types of in-mold cavity sensors were installed (SSB01KN08X06H and SSB01KN08X08H; Futaba, Mobara, Japan).

Figure 4 illustrates the locations of each sensor. To study the overall flow state of the melt and its response to the quality indices, seven pressure sensors were installed in the mold, with one at the sprue, one in front of the gate, one near the gate, two at the center of the cavity, and two far from the gate. The polymer melt was assumed to advance in laminar flow because of its typical fountain flow behavior; the ratio of the flow direction distance to the average thickness was also high (124), and pressure change along the thickness direction was ignored.

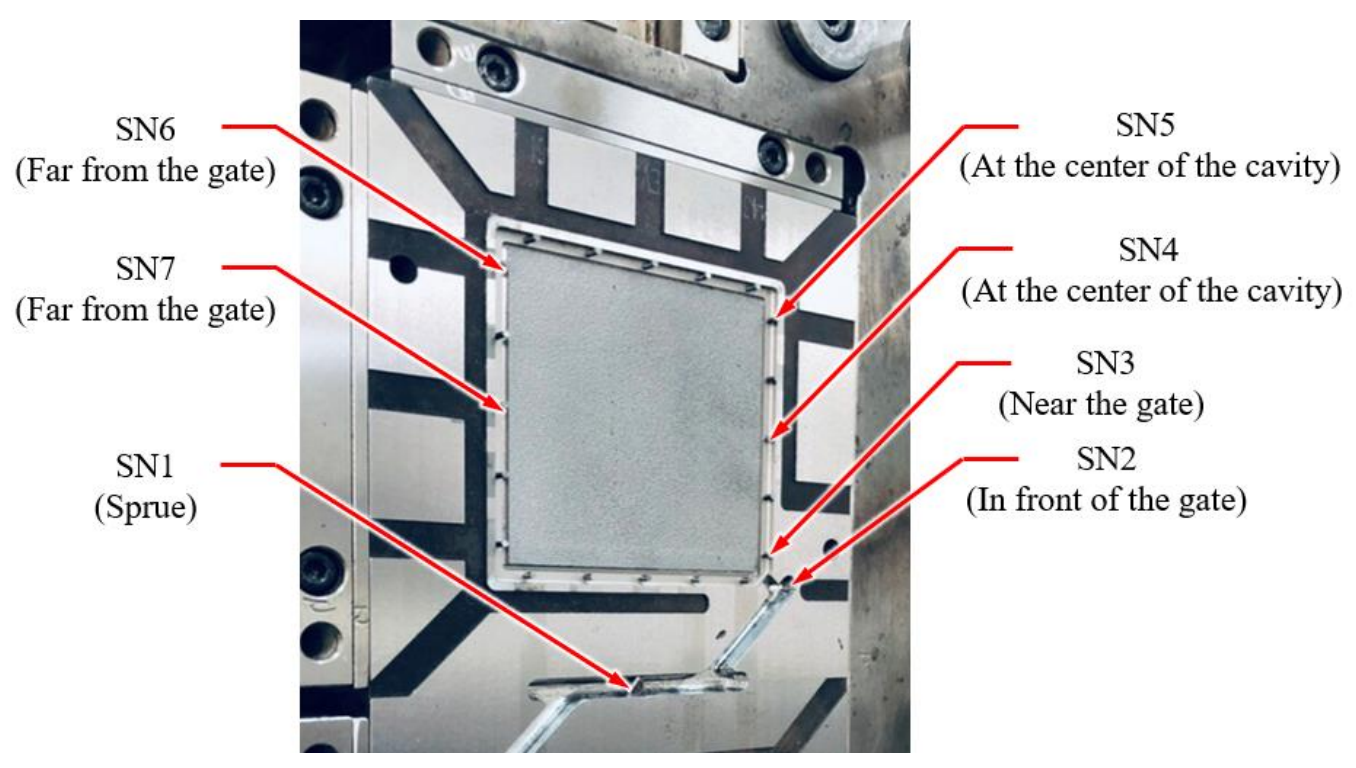

Fig. 4 Positions of the pressure sensors installed in the cavity [16]

Table 1 lists the process parameter settings in the injection molding experiment. For this experiment, a two-factor full factorial method was adopted, in which the injection speed range 
was adjusted from 40 to $120 \mathrm{~mm} / \mathrm{s}$ and the first-stage holding pressure varied from 50 to 100 $\mathrm{MPa}$; data were collected four to eight times with the same parameters. At each shot, a system pressure curve and seven cavity pressure curves (SN1-SN7) were recorded. The system pressure curve was used to obtain two quality indices, namely the $P h_{\text {index }}$ and $P I_{\text {index }}$. The seven cavity pressure curves (SN1-SN7) were used to obtain the $P p_{\text {index }}$, and four curves (SN4-SN7) produced by sensors installed far from the gate were used to obtain the $P r_{\text {index }}$. A total of 445 subexperiments were conducted, each comprising 11 quality indices. These quality indices were used for the input data of the MLP model.

Table 1 Molding parameter setting

\begin{tabular}{|c|c|c|c|}
\hline \multicolumn{2}{|l|}{ Item } & Unit & Parameters \\
\hline \multicolumn{2}{|l|}{ Melt temperature } & ${ }^{\circ} \mathrm{C}$ & 205 \\
\hline \multicolumn{2}{|l|}{ Mold temperature } & ${ }^{\circ} \mathrm{C}$ & 60 \\
\hline \multicolumn{2}{|l|}{ Backpressure } & $\mathrm{MPa}$ & 4.5 \\
\hline \multicolumn{2}{|l|}{ Clamping force } & Tons & 70 \\
\hline \multicolumn{2}{|c|}{ Decompression on stroke } & $\mathrm{mm}$ & 10 \\
\hline \multicolumn{2}{|c|}{ Holding speed limit } & $\mathrm{mm} / \mathrm{s}$ & 80 \\
\hline \multicolumn{2}{|c|}{ V--P switchover position } & $\mathrm{mm}$ & 12.45 \\
\hline \multicolumn{2}{|c|}{ Cooling time } & $\mathrm{s}$ & 16 \\
\hline \multirow{3}{*}{ Holding pressure } & 1st stage & $\mathrm{MPa}$ & $40,50,60,70,80,90,100$ \\
\hline & 2nd stage & $\mathrm{MPa}$ & 5 \\
\hline & 3rd stage & $\mathrm{MPa}$ & 15 \\
\hline \multirow{3}{*}{ Holding time } & 1st stage & $\mathrm{S}$ & 1 \\
\hline & 2nd stage & $\mathrm{s}$ & 4 \\
\hline & 3rd stage & $\mathrm{s}$ & 5 \\
\hline \multicolumn{2}{|l|}{ Injection speed } & $\mathrm{mm} / \mathrm{s}$ & $40,50,60,70,80,90,100,110,120$ \\
\hline
\end{tabular}

Figure 5 depicts the width measurement position, measured using a three-coordinate measurement machine (CRYSTA-Apex S700; Mitutoyo, Kawasaki, Japan). The quality is represented by the width of the IC tray geometry. To classify the quality of injection molded parts, we converted the measured geometrical width into multiple grades. We subsequently aggregated the data into five grades evenly spaced between the minimum and maximum values in width, which were used as the output data of the MLP model. 


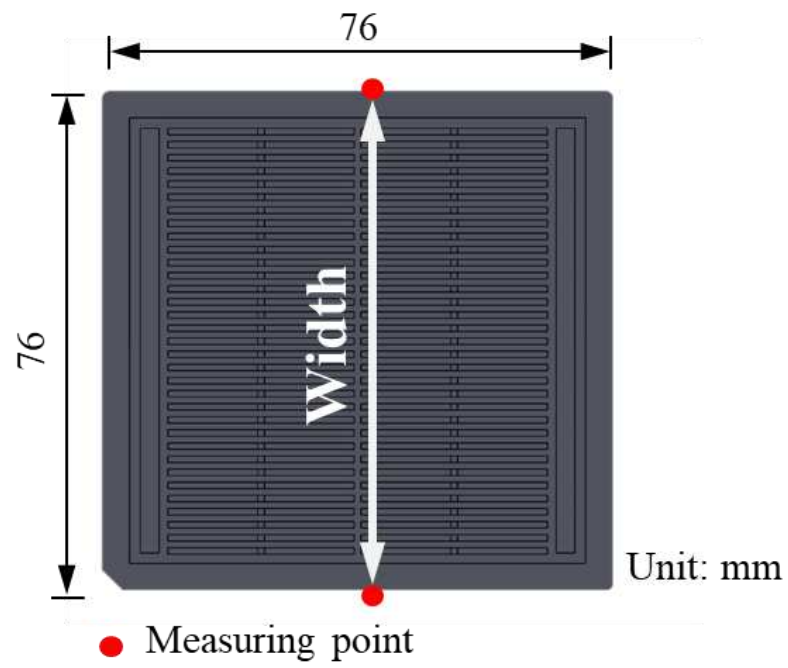

Fig. 5 IC tray and its width quality measurement location

\subsection{Experimental procedure}

A flowchart of the entire process operation is presented in Fig. 6, including data preprocessing, hyperparameter adjustment, and data training. A detailed description is outlined as follows:

(1) Pressure signal extraction and quality measurement: The pressure curve of the molding process was obtained through the in-mold sensors, and a three-dimensional measuring instrument was used to measure its width value, thereby establishing a pressure history and width value database.

(2) Outlier filtering: The standard score was used as the basis for judgment, with the $z$ value of 1.78 selected as the standard to eliminate outlier values under the same molding parameters. Among them, the 1.78 standard score is equivalent to retaining $92.5 \%$ of the data volume [17].

(3) Quality indices transformation: The extracted in-mold pressure curve was converted into quality indices for subsequent machine learning.

(4) Data normalization: The size and dimension of the input values have distinct effects on data convergence. Processing prior to data normalization reduces the adverse effects of the different dimension values on the convergence results [27]. We used max-min 
normalization in this experiment.

(5) MLP model training: We employed the SGD and SGDM as optimizers in this experiment. These were applied to a fixed-architecture MLP for data training. The nodes of the input, first hidden, second hidden, and output layers were 11, 50, 25, and 5, respectively.

(6) Hyperparameter adjustment: This experiment focused on the changes of five different activation functions, momentum, and learning rate to assess the results of learning accuracy and convergence behavior. The range of momentum was 0.1 to 0.9 and the range of learning rate was $10^{-5}$ to $10^{-1}$ and 0.1 to 0.9 . A full factorial experimental design was employed in this study.

(7) Training and testing results: The effect of momentum and learning rate on accuracy was assessed by recording the training and verification learning accuracy and efficiency, respectively.

(8) Performance index calculation: To generate statistical trends and enhance physical interpretability, five trainings were conducted under the same hyperparameter settings; the learning rate and efficiency were recorded and the average calculated for subsequent evaluation. 


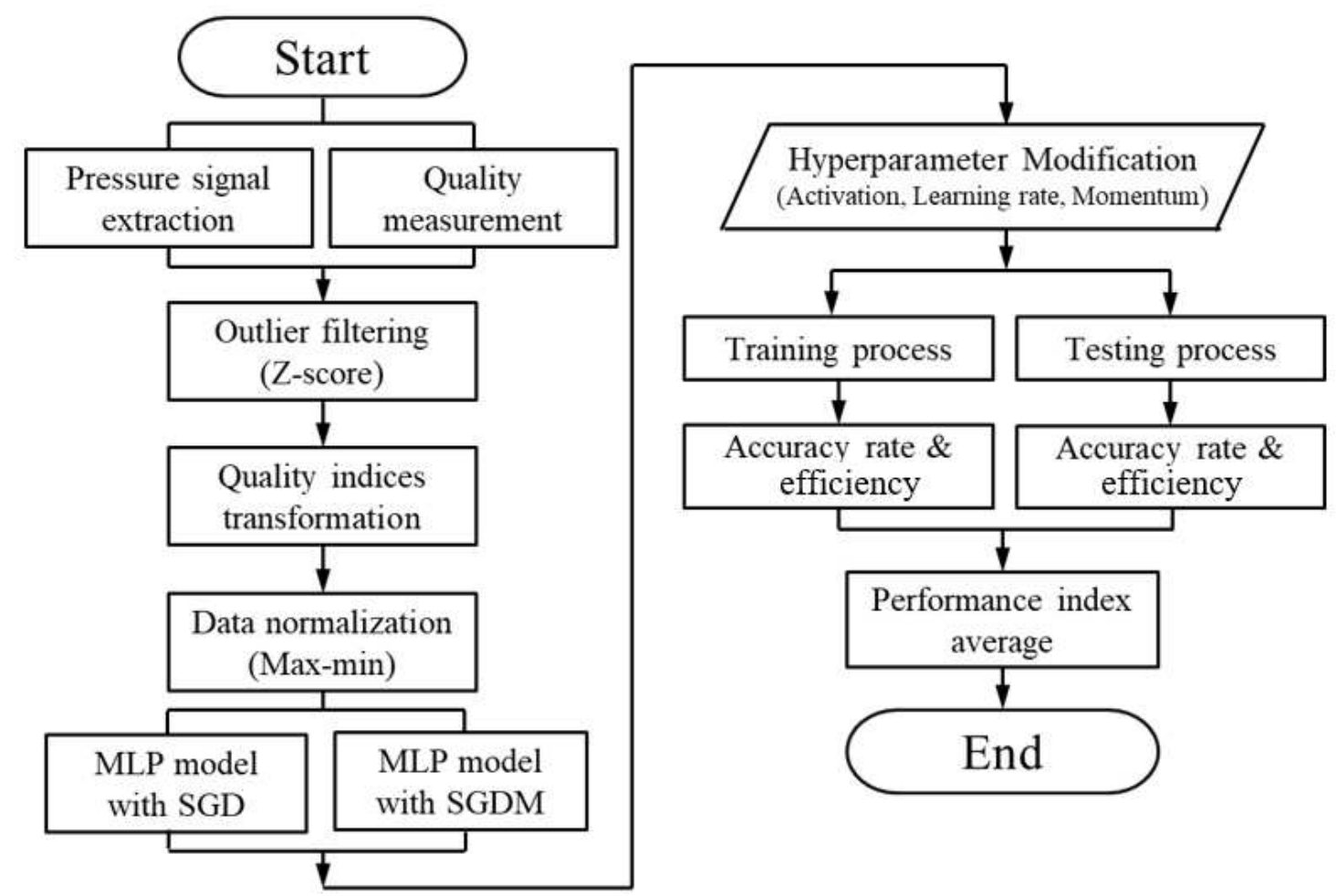

Figure 6. Flowchart of model training.

\subsection{MLP model and hyperparameter settings}

Table 2 describes the MLP model used in this experiment and the corresponding hyperparameter settings. The number of nodes in the input layer, two hidden layers, and output layer of the MLP model was 11,50/25, and 5, respectively. The number of training and testing datasets was 337 and 84, respectively, and the two optimized solvers SGD and SGDM were used for training with 7200 iterations. Both solvers set the learning rate value, and the SGDM adjusted the momentum value. The adjustment range of momentum was 0 to 0.9 . We classified two groups, Group 1 and Group 2, to explore the influence of learning rate dimension on training accuracy. The training hardware specifications used in this experiment are detailed in Table 3. 
Table 2 MLP model and hyperparameter settings

\begin{tabular}{lll}
\hline Hyperparameter & & Values \\
\hline Number neuron of & input layer & 11 \\
& $1^{\text {st }}$ hidden layers & 50 \\
& $2^{\text {nd }}$ hidden layers & 25 \\
& output layer & 5 \\
\hline Mini batch size & & 20 \\
\hline Learning rate $(\alpha)$ & Group 1 & $10^{-5}, 10^{-4}, 10^{-3}, 10^{-2}, 10^{-1}$ \\
& Group 2 & $0.1,0.2,0.3,0.4,0.5,0.6,0.7,0.8,0.9$ \\
\hline Momentum $(\beta)$ & & $0,0.1,0.2,0.3,0.4,0.5,0.6,0.7,0.8,0.9$ \\
Iteration & & 7200 \\
Activation function & & Sigmoid, ReLU, Tanh, Leaky ReLU, ELU \\
No. training dataset & & 337 \\
No. testing dataset & & 84 \\
\hline
\end{tabular}

Table 3 Hardware specifications

\begin{tabular}{ll}
\hline Hardware & Specification \\
\hline Central processing unit & AMD R9 3900x \\
Graphic processing unit & GIGABITE GeFORCE RTX 3090 \\
Memory & 32 GB \\
Training software & Matlab r2020b \\
\hline
\end{tabular}

\section{Results and discussion}

To explore the difference between conventional SGD and SGDM, the model was trained through adjustment of the activation function, learning rate $(\alpha)$, and momentum $(\beta)$ to capture its training and test accuracy. The effects of five activation functions (Sigmoid, Tanh, ReLU, Leaky ReLU, and ELU) on the learning rate was also analyzed. The $\alpha$ was divided into Group 1 , ranging from $10^{-5}$ to $10^{-1}$, and Group 2 , ranging from 0.1 to 0.9 , for experimental adjustment. Finally, the $\beta$ was set in a range of 0.1 to 0.9 .

\subsection{Group 1 with zero momentum}

The goal was to compare the effect of learning rate adjustment on the training accuracy of the MLP by using five activation functions in the SGD. We evaluated the training rate set for 
various activation functions following 7200 iterations. Figure 7(a) and 7(b) illustrate the training and testing accuracy when the learning rate was set to $10^{-5}$ and $10^{-4}$, respectively, both of which proved inefficient $(14 \%-65 \%)$ and are not recommended for training MLP models. However, when the learning rate was set to $10^{-3}$, the ReLU and ELU activation functions exhibited high training and testing accuracy (over 90\%) and rapid convergence. When the learning rate was increased to $10^{-1}$, the accuracy of all activation functions reached over $90 \%$. Among them, the Tanh and ELU functions had the most rapid convergence speed at a learning rate of $10^{-1}$, although the accuracy of these activation functions exceeded $90 \%$ in only 151 iterations (Table 4). At a low learning rate of $10^{-3}$, the ELU and ReLU reached a learning accuracy of over $90 \%$ in 6193 and 6332 iterations, respectively, indicating effective convergence in model training. A learning rate of $10^{-1}$ provided effective training and convergence results in relation to learning accuracy (Table 4). For the Sigmoid function, the highest training accuracy was observed at a learning rate of $10^{-1}$, but 1177 iterations were required before its training accuracy exceeded $90 \%$, which was the slowest rate of increase of all activation functions. 


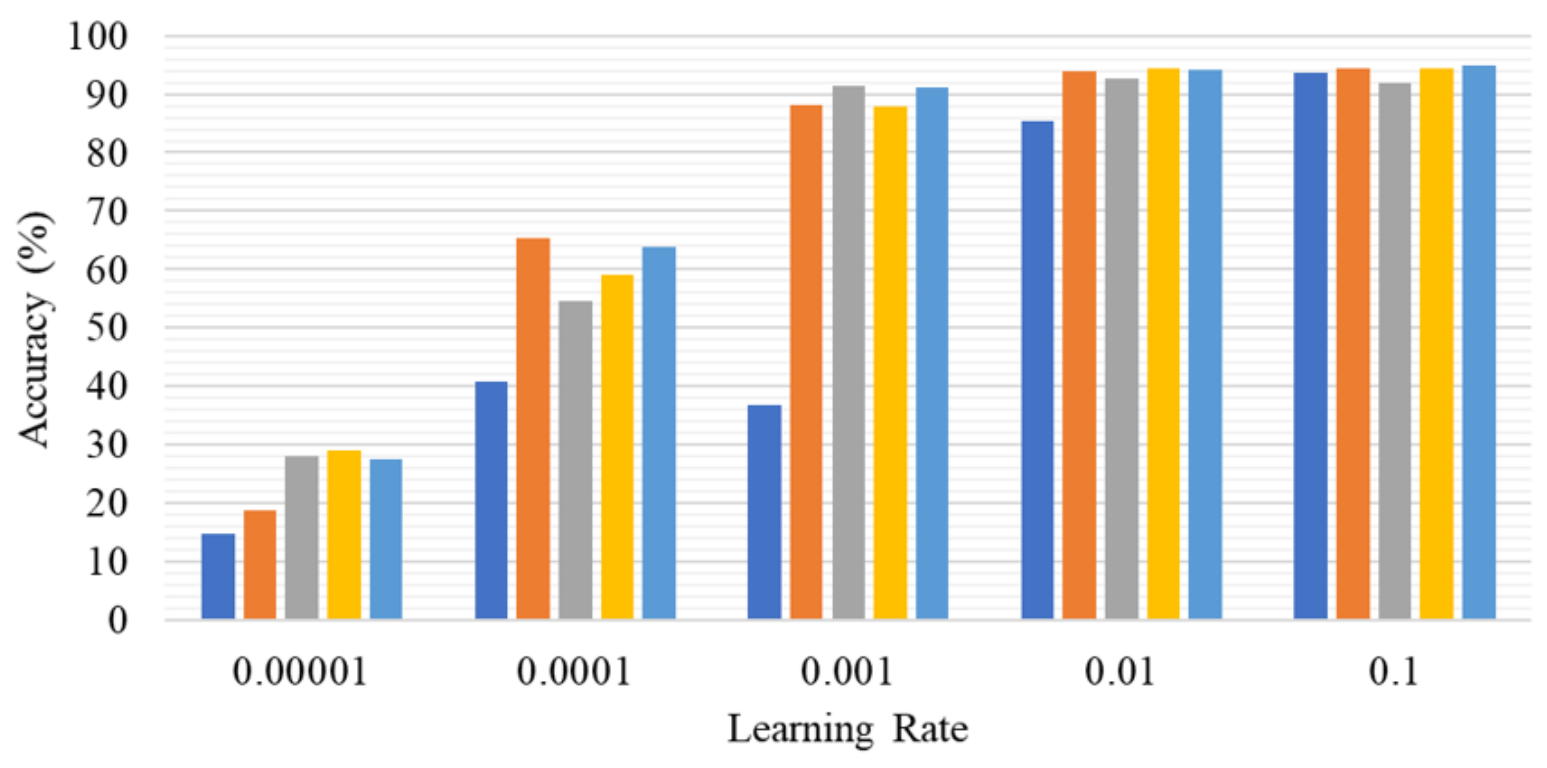

घigmoid $\quad$ Tanh $\square$ ReLU $\square$ Leaky ReLU $\square$ ELU

(a) Training accuracy

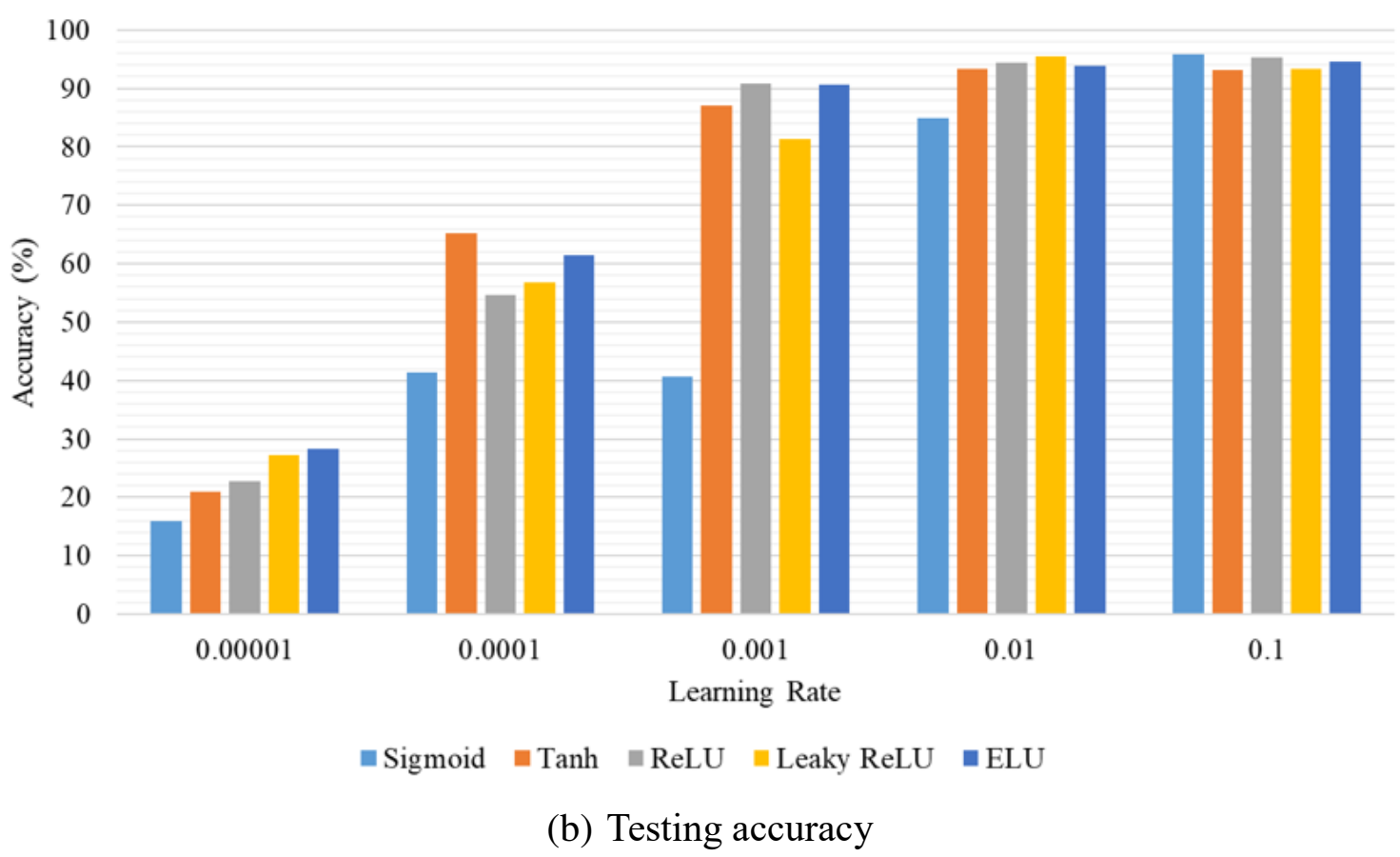

Fig. 7 Accuracy of Group 1 with zero momentum after 7200 iterations 
Table 4 Number of iterations required for Group 1 with zero momentum to exceed a training rate of $90 \%$.

\begin{tabular}{lrrrrr}
\hline Activation & \multicolumn{5}{c}{ Learning rate } \\
\cline { 2 - 6 } Function & $10^{-5}$ & $10^{-4}$ & $10^{-3}$ & $10^{-2}$ & $10^{-1}$ \\
\hline Sigmoid & - & - & - & - & 1177 \\
Tanh & - & - & - & 815 & 151 \\
ReLU & - & - & 6332 & 1139 & 417 \\
Leaky ReLU & - & - & - & 833 & 239 \\
ELU & - & - & 6193 & 675 & 151 \\
\hline
\end{tabular}

\subsection{Group 2 with zero momentum}

Different activation functions require an appropriate learning rate to achieve high learning accuracy. A learning rate of $10^{-1}$ enabled all five activation functions to obtain the optimal model learning performance. The learning rate was thus increased beyond $10^{-1}$ to investigate its performance in terms of accuracy, convergence, and stability.

Figure 8 and Table 5 present the testing accuracy of Group 2 with zero momentum after 7200 iterations. Except for the ELU, the other activation functions achieved high training accuracy above $90 \%$. The Sigmoid function outperformed the others, with an average testing accuracy of $93.8 \%$; its test accuracy reached $95.8 \%$ when the learning rate was 0.1 , followed by $95.1 \%$ when the learning rate was 0.7 . For the Tanh, ReLU, and Leaky ReLU functions, their learning rates exceeded $91 \%$ for various learning rates set within the range of 0.1 to 0.9 . By contrast, the ELU function achieved a test accuracy of over $90 \%$ at a learning rate set in the range of 0.1 to 0.6 but performed ineffectively at a learning rate in the range of 0.7 to 0.9 . 


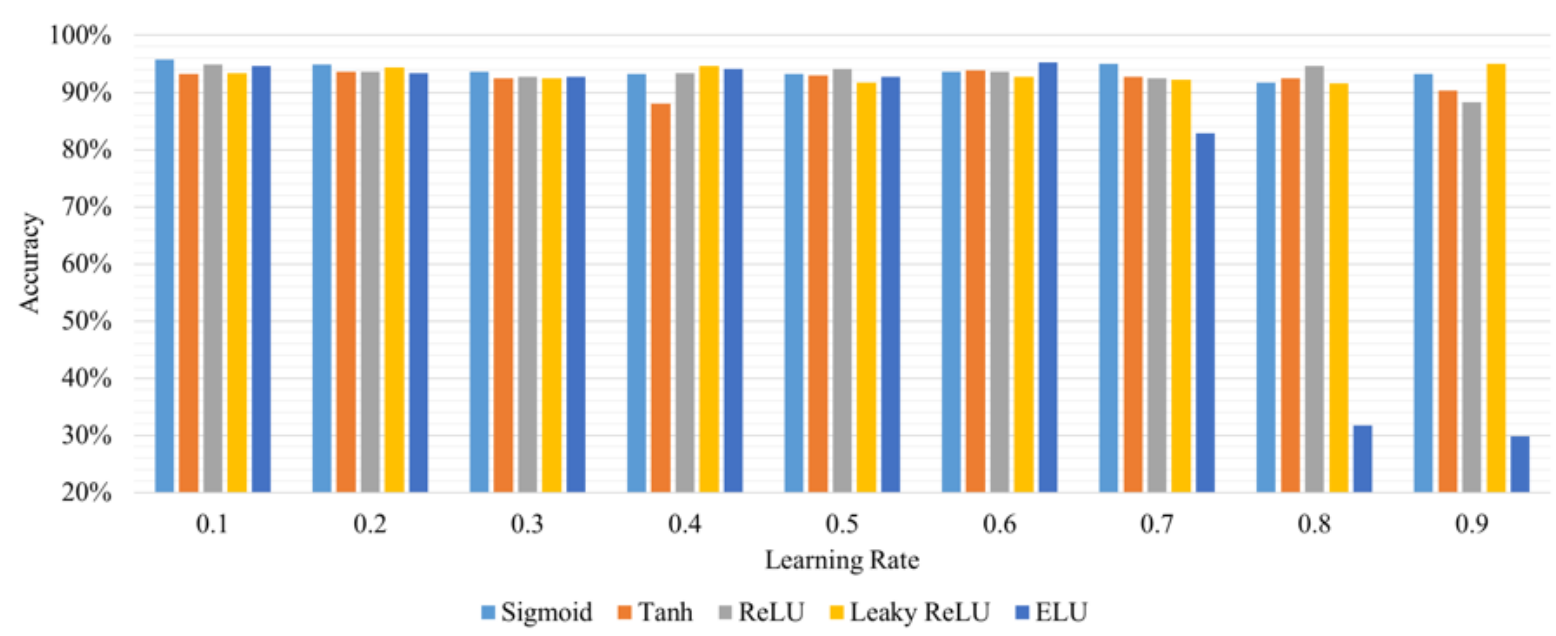

Fig. 8 Testing accuracy of Group 2 with zero momentum under 7200 iterations

Table 5 Testing accuracy of Group 2 with zero momentum under 7200 iterations

\begin{tabular}{lrrrrrrrrrr}
\hline Activation & \multicolumn{10}{c}{ Learning rate } \\
\cline { 2 - 11 } Function & 0.1 & 0.2 & 0.3 & 0.4 & 0.5 & 0.6 & 0.7 & 0.8 & 0.9 & Avg. \\
\hline Sigmoid & 95.8 & 94.8 & 93.7 & 93.2 & 93.2 & 93.7 & 95.1 & 91.8 & 93.2 & 93.8 \\
Tanh & 93.2 & 93.7 & 92.5 & 88.0 & 92.9 & 93.9 & 92.7 & 92.5 & 90.4 & 92.2 \\
ReLU & 94.8 & 93.7 & 92.7 & 93.4 & 94.1 & 93.7 & 92.5 & 94.6 & 88.2 & 93.1 \\
Leaky ReLU & 93.4 & 94.4 & 92.5 & 94.6 & 91.8 & 92.7 & 92.2 & 91.5 & 95.1 & 93.1 \\
ELU & 94.6 & 93.4 & 92.7 & 94.1 & 92.7 & 95.3 & 82.8 & 31.8 & 29.9 & 78.6 \\
\hline & & & \multicolumn{1}{c}{ Unit of Accuracy: \% }
\end{tabular}

In addition to the stability and quality of the learning accuracy, learning efficiency is critical to reducing computation time. Figure 9 and Table 6 describe the number of iterations required for Group 2 with zero momentum to exceed a training rate of $90 \%$. To further analyze the relationship between the learning rate and number of iterations required to exceed $90 \%$, the correlation coefficients are listed in Table 7. As described in Fig. 9, the activation functions Tanh, ReLU, Leaky ReLU, and ELU and number of iterations required to exceed a 90\% learning rate were positively correlated with the learning rate, with correlation coefficients of $0.82,0.74,0.84$, and 0.88 , respectively. These strong positive correlations indicated that an increase in the learning rate may slow convergence speed, thus requiring more iterations. Moreover, even at the highest learning rate, the ReLU and ELU functions could not achieve 90\% training accuracy. Conversely, the Sigmoid function had a strong negative correlation coefficient $(-0.84)$, demonstrating that effective convergence can be obtained with an increased 
learning rate. Notably, with a learning rate of 0.8 , only 271 iterations were required to obtain a learning accuracy of over $90 \%$.

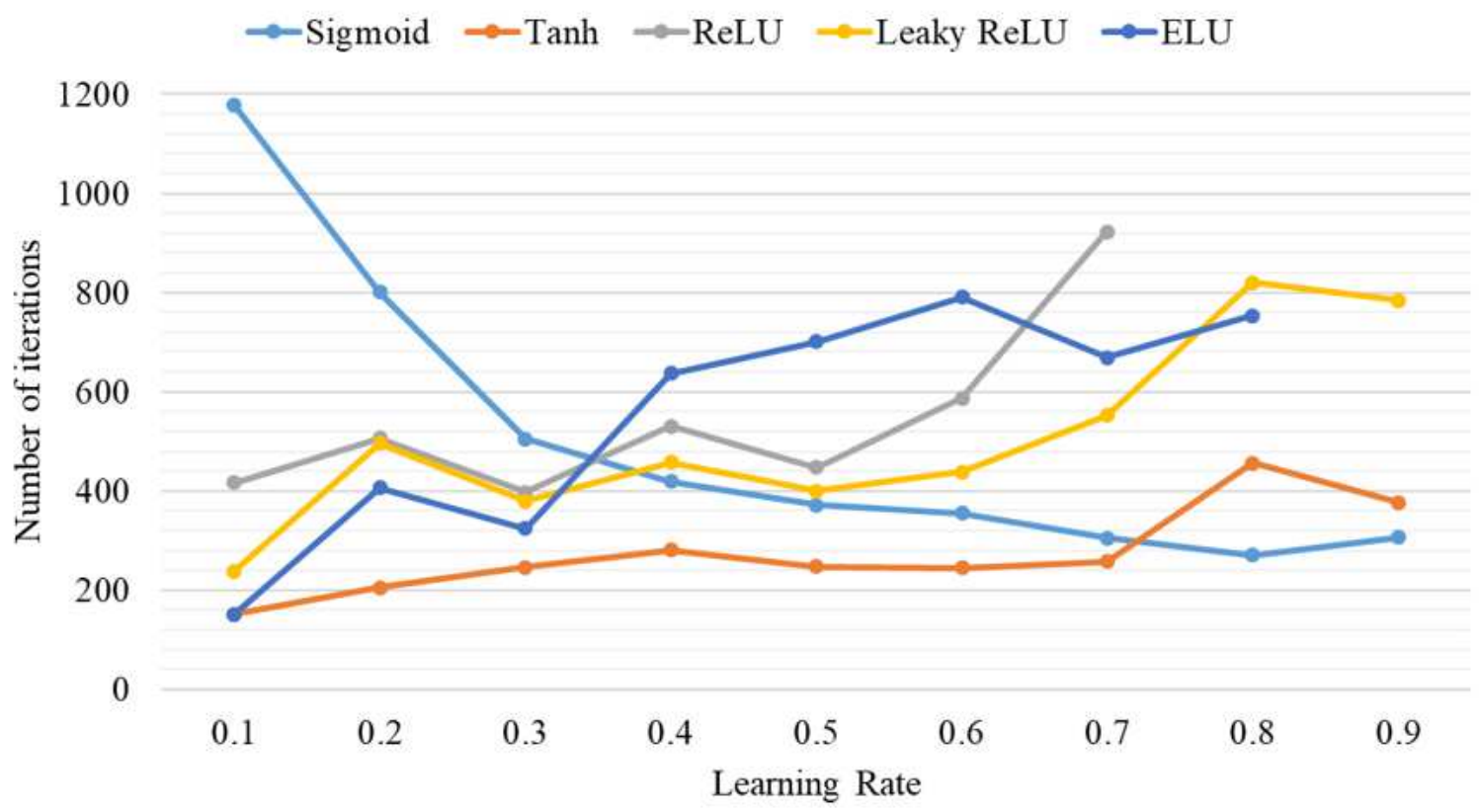

Fig. 9 Number of iterations required for Group 2 with zero momentum to achieve 90\% training accuracy

Table 6 Number of iterations required for Group 2 with zero momentum to achieve $90 \%$ training accuracy

\begin{tabular}{lrrrrrrrrr}
\hline Activation & \multicolumn{8}{c}{ Learning rate } \\
\cline { 2 - 10 } Function & 0.1 & 0.2 & 0.3 & 0.4 & 0.5 & 0.6 & 0.7 & 0.8 & 0.9 \\
\hline Sigmoid & 1177 & 801 & 505 & 419 & 372 & 355 & 305 & 271 & 307 \\
Tanh & 151 & 205 & 246 & 281 & 247 & 245 & 258 & 456 & 377 \\
ReLU & 417 & 506 & 397 & 531 & 448 & 587 & 923 & - & - \\
Leaky ReLU & 239 & 496 & 380 & 458 & 400 & 439 & 552 & 820 & 784 \\
ELU & 151 & 407 & 324 & 637 & 701 & 790 & 669 & 754 & - \\
\hline & & & & & - : less than $90 \%$ at 7200 iterations
\end{tabular}


Table 7 Correlation coefficients of the learning rate and number of iterations (Group 2 with zero momentum)

\begin{tabular}{llllll}
\hline & \multicolumn{5}{c}{ Activation function } \\
\cline { 2 - 6 } & Sigmoid & Tanh & ReLU & Leaky ReLU & ELU \\
\cline { 2 - 6 } $\begin{array}{l}\text { Pearson's } \\
\text { correlation } \\
\text { coefficient }\end{array}$ & -0.84 & 0.82 & 0.74 & 0.84 & 0.88 \\
\hline
\end{tabular}

Although a high learning rate generally promotes rapid convergence, the stability of the combined learning rate and activation function must be monitored in model learning. This study evaluated the stability through calculation of training accuracy deviation when the learning rate exceeded $90 \%$ in model training. Figure 10 illustrates that a learning rate in the range of 0.1 to 0.5 resulted in a training accuracy deviation of approximately 7\% for Group 2 with zero momentum. By contrast, when the learning rate was in the range of 0.6 to 0.9 , the training accuracy deviation of the ReLU and ELU functions increased considerably, indicating model instability and a high correlation with the learning rate. The Sigmoid activation function maintained a deviation of less than $5 \%$ under various learning rates, demonstrating a low oscillation phenomenon at a high learning rate and the ability to effectively maintain convergence (Table 8).

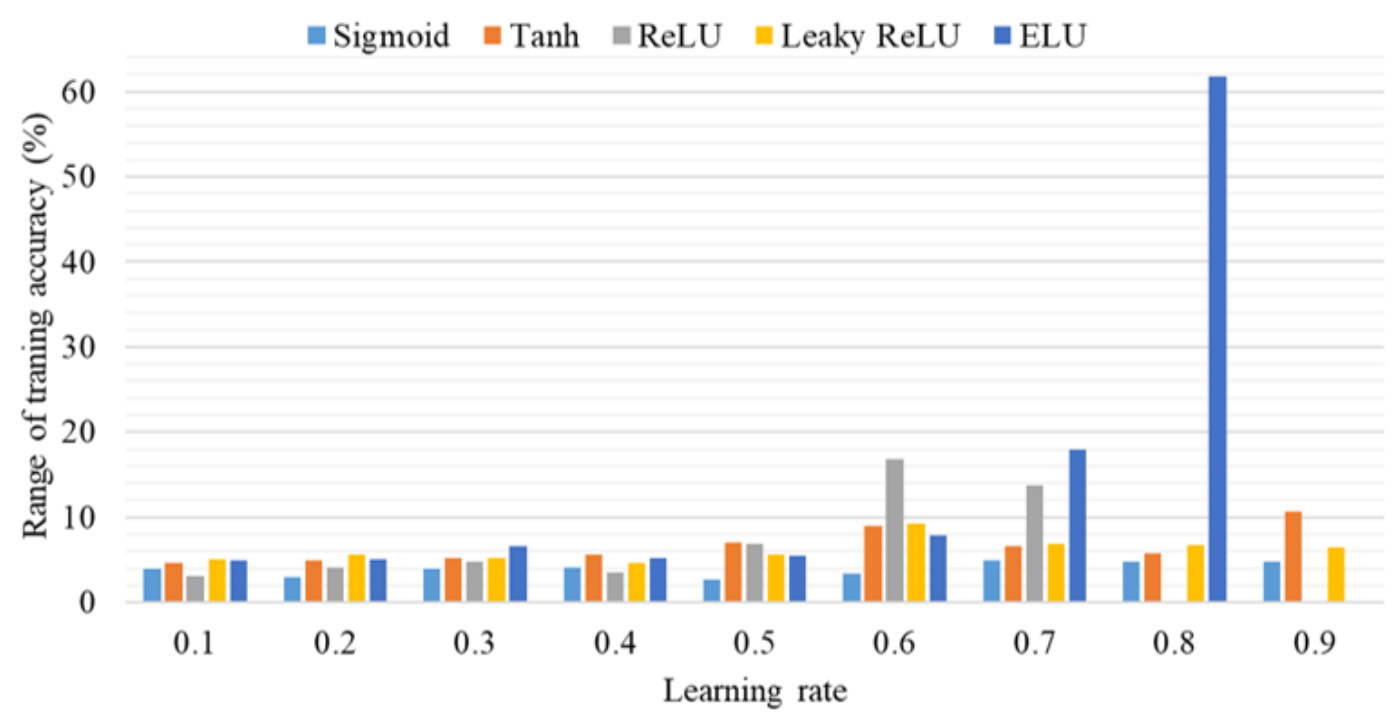

Fig. 10 Range of learning accuracy for Group 2 with zero momentum after exceeding 90\% 
Table 8 Range of learning accuracy for Group 2 with zero momentum after exceeding $90 \%$

\begin{tabular}{lrrrrrrrrrr}
\hline Activation & \multicolumn{10}{c}{ Learning rate } \\
\cline { 2 - 12 } Function & 0.1 & 0.2 & 0.3 & 0.4 & 0.5 & 0.6 & 0.7 & 0.8 & 0.9 & Avg. \\
\hline Sigmoid & 3.9 & 2.9 & 3.9 & 4.1 & 2.7 & 3.3 & 4.9 & 4.7 & 4.8 & 3.9 \\
Tanh & 4.6 & 5.0 & 5.3 & 5.7 & 7.0 & 8.9 & 6.6 & 5.7 & 10.6 & 6.6 \\
ReLU & 3.1 & 4.1 & 4.8 & 3.5 & 6.8 & 16.8 & 13.7 & - & - & 7.6 \\
Leaky ReLU & 5.0 & 5.6 & 5.2 & 4.7 & 5.6 & 9.2 & 6.9 & 6.8 & 6.4 & 6.2 \\
ELU & 4.9 & 5.0 & 6.7 & 5.2 & 5.5 & 7.9 & 17.9 & 61.8 & - & 14.4 \\
\hline & & & & & & & 7 & Unit of Accuracy: \% \\
& & & & & & & - : less than $90 \%$ at 7200 iterations
\end{tabular}

\subsection{Effect of momentum on convergence}

This experiment employed momentum acceleration in the SGDM method to explore the influence of momentum on modeling accuracy. Figure 11 and Table 9 present the testing accuracy of the SGDM after 7200 iterations at a learning rate of $0.1(\alpha=0.1)$. The introduction of momentum did not substantially improve the testing accuracy of the five activation functions; the testing accuracy of the ELU and ReLU functions actually decreased with the addition of momentum. Table 10 lists the correlation coefficients of momentum and the number of iterations required to achieve $90 \%$ testing accuracy. The correlation coefficients of the Sigmoid, Tanh, ReLU, Leaky ReLU, and ELU functions were $-0.14,-0.3,0.62,0.23$, and 0.41 , respectively, indicating that momentum was not strongly correlated with the number of iterations..

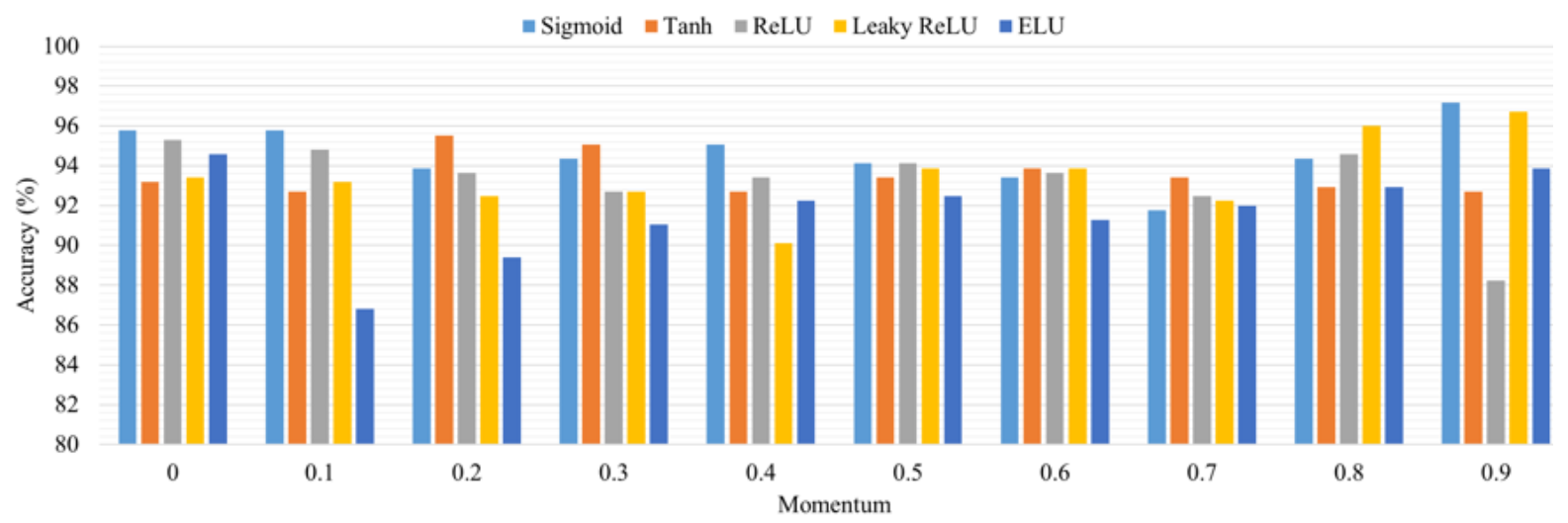

Fig. 11 Testing accuracy of the SGDM $(\alpha=0.1)$ under 7200 iterations 
Table 9 Testing accuracy of the SGDM $(\alpha=0.1)$ under 7200 iterations

\begin{tabular}{lrrrrrrrrrr}
\hline Activation & \multicolumn{10}{c}{ Momentum } \\
\cline { 2 - 11 } Function & 0.0 & 0.1 & 0.2 & 0.3 & 0.4 & 0.5 & 0.6 & 0.7 & 0.8 & 0.9 \\
\hline Sigmoid & 95.8 & 95.8 & 93.9 & 94.4 & 95.1 & 94.1 & 93.4 & 91.8 & 94.4 & 97.2 \\
Tanh & 93.2 & 92.7 & 95.5 & 95.1 & 92.7 & 93.4 & 93.9 & 93.4 & 92.9 & 92.7 \\
ReLU & 95.3 & 94.8 & 93.7 & 92.7 & 93.4 & 94.1 & 93.7 & 92.5 & 94.6 & 88.2 \\
Leaky ReLU & 93.4 & 93.2 & 92.5 & 92.7 & 90.1 & 93.9 & 93.9 & 92.2 & 96.0 & 96.7 \\
ELU & 94.6 & 86.8 & 89.4 & 91.1 & 92.2 & 92.5 & 91.3 & 92.0 & 92.9 & 93.9 \\
\hline & & & & & & & & Unit of Accuracy: \%
\end{tabular}

Table 10 Correlation coefficients of momentum and the number of iterations $(\alpha=0.1)$

\begin{tabular}{llllll}
\hline & \multicolumn{5}{c}{ Activation function } \\
\cline { 2 - 6 } & Sigmoid & Tanh & ReLU & Leaky ReLU & ELU \\
\cline { 2 - 6 } $\begin{array}{l}\text { Pearson's } \\
\text { correlation } \\
\text { coefficient }\end{array}$ & -0.14 & -0.3 & -0.62 & 0.54 & 0.41 \\
\hline
\end{tabular}

Figure 12 and Table 11 detail the number of iterations required for model learning to achieve a 90\% training rate, and Table 12 lists the correlation coefficients of the number of iterations and momentum. The Sigmoid function, with a correlation coefficient of -0.99 , was the most effective in relation to changes in momentum. For momentum change from 0.1 to 0.9 , the number of iterations required to reach a 90\% training rate was markedly reduced from 1193 to 210 (improvement rate: $82.4 \%$ ). The Tanh function had a strong negative correlation with momentum (correlation coefficient: -0.79 ), and the number of iterations required to achieve a $90 \%$ training rate was significantly reduced from 151 to 98 (improvement rate: $35.1 \%$ ). By contrast, the ReLU, Leaky ReLU, and ELU functions exhibited minimal correlation with momentum; thus, momentum changes did not affect the learning efficiency. 


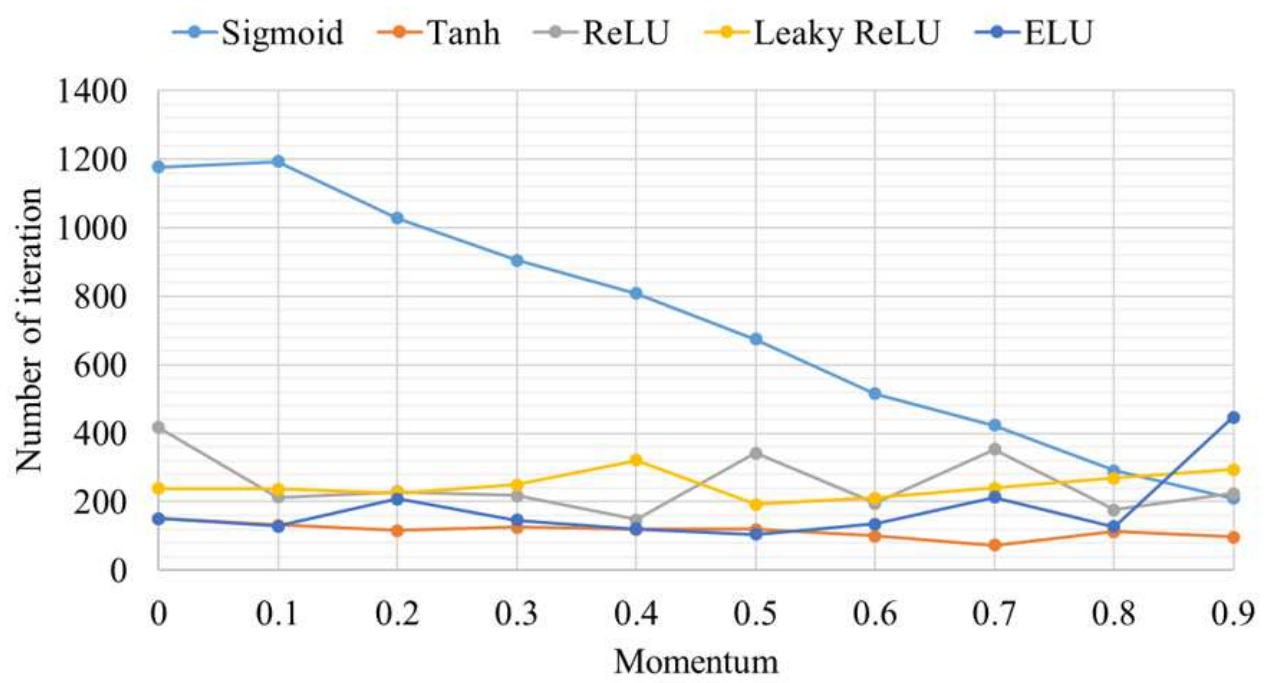

Fig. 12 Number of iterations required for the SGDM for Group 2 with momentum to achieve $90 \%$ training accuracy $(\alpha=0.1)$

Table 11 Number of iterations required for the SGDM for Group 2 with momentum to achieve $90 \%$ training accuracy $(\alpha=0.1)$

\begin{tabular}{lrrrrrrrrrr}
\hline Activation & \multicolumn{10}{c}{ Momentum } \\
\cline { 2 - 11 } Function & 0.0 & 0.1 & 0.2 & 0.3 & 0.4 & 0.5 & 0.6 & 0.7 & 0.8 & 0.9 \\
\hline Sigmoid & 1177 & 1193 & 1027 & 905 & 809 & 675 & 516 & 423 & 291 & 210 \\
Tanh & 151 & 133 & 117 & 126 & 119 & 119 & 101 & 73 & 114 & 98 \\
ReLU & 417 & 214 & 229 & 219 & 148 & 342 & 196 & 353 & 177 & 225 \\
Leaky ReLU & 239 & 238 & 226 & 250 & 322 & 192 & 212 & 241 & 269 & 295 \\
ELU & 151 & 129 & 208 & 146 & 122 & 106 & 135 & 213 & 127 & 448 \\
\hline
\end{tabular}

Table 12 Correlation coefficients of the number of iterations and momentum $(\alpha=0.1)$

\begin{tabular}{llllll}
\hline & \multicolumn{5}{c}{ Activation function } \\
\cline { 2 - 6 } & Sigmoid & Tanh & ReLU & Leaky ReLU & ELU \\
\cline { 2 - 6 } $\begin{array}{l}\text { Pearson's } \\
\text { correlation } \\
\text { coefficient }\end{array}$ & -0.99 & -0.79 & -0.26 & 0.26 & 0.48 \\
\hline
\end{tabular}

\subsection{Hyperparameters optimization}

This analysis revealed that hyperparameter settings affect the accuracy and efficiency of model training. Through optimization of hyperparameter settings, model performance can be improved. Table 13 presents the two sets of hyperparameter settings, which were initially based on experience, with the later optimization process based on the recommendations in Section 4.1 
to 4.3 . The testing accuracy of the initial and optimized settings was $87.4 \%$ and $97.2 \%$, respectively. Therefore, adjustment of the learning rate and momentum can vastly improve the accuracy of model testing (up to $9.8 \%$ improvement rate). In regards to the training performance efficiency, the number of iterations required for the initial and optimized settings to achieve $80 \%$ training accuracy was 5680 and 210 , respectively; therefore, the optimized hyperparameter settings markedly reduced training time. Overall, the experimental results demonstrated that optimizing hyperparameter settings not only improves the accuracy of model testing but also considerably reduces training time.

Table 13 Comparison of various hyperparameter settings

\begin{tabular}{lll}
\hline Hyperparameter & Initial & Optimized \\
\hline Learning rate & 0.01 & 0.1 \\
Momentum & 0.1 & 0.9 \\
Max. iteration & 7200 & 7200 \\
Activation function & Sigmoid & Sigmoid \\
\hline Testing accuracy & $87.4 \%$ & $97.2 \%$ \\
Iterations to reach $80 \%$ training accuracy & 5,680 & 210 \\
\hline
\end{tabular}

\section{Conclusion}

This study examined the influence of hyperparameters on the accuracy and convergence of MLP model training. In this investigation, IC tray injection molding cavity pressure curves were measured using sensors and converted to normalized quality indices to serve as the input data for model training; the part size was used as the output data. The MLP architecture comprised 11 nodes in the input layer, 75 and 50 nodes in the first and second hidden layer, respectively, and 5 nodes in the output layer. In addition, this study provided a comparison of the SGD and SGDM optimizers, a comparison of five activation functions (Sigmoid, ReLU, Tanh, Leaky ReLU, and ELU), and an evaluation of learning rate and momentum adjustment and its effects on the accuracy and efficiency of model training. The results are summarized as follows:

(1) Regarding the change of learning rate in the SGD algorithm without momentum, when the 
learning rate was altered from $10^{-5}$ to $10^{-1}$, functions with a learning rate of $10^{-1}$ performed most effectively, with the learning rate exceeding $90 \%$. The number of iterations required for the training accuracy of the five excitation functions to achieve more than $90 \%$ are as follows: Sigmoid, 1177; Tanh, 151; ReLU, 417; Leaky ReLU, 239; and ELU, 151. Therefore, the Tanh and ELU excitation functions combined with the SGD can obtain rapid convergence, whereas the Sigmoid function was relatively slow, with a 7.8 times difference in the convergence rate.

(2) With the introduction of momentum ranging from 0.1 to 0.9 , the Sigmoid function achieved an average training accuracy of $93.8 \%$, outperforming the other five functions. In addition, its strong correlation with momentum was reflected in the rapid convergence rate. For instance, when the learning rate was 0.8 , only 271 iterations were required to obtain a learning accuracy over $90 \%$.

(3) When the learning rate was between 0.1 and 0.5 , the training accuracy ranges of the five functions were all within $7 \%$. When the learning rate was between 0.6 and 0.9 , only the training accuracy of the Sigmoid activation function remained below 5\%, indicating its stability in MLP model training.

(4) For the momentum change (0.1-0.9) in the SGDM algorithm under a learning rate of 0.1 , the testing accuracy of the five functions did not improve substantially after 7200 iterations. However, the correlation coefficient of the number of iterations and momentum of the Sigmoid function was -0.99 , indicating a strong negative correlation. For momentum change from 0.1 to 0.9 , the number of iterations required to reach a $90 \%$ training rate reduced from 1193 to 210 (improvement rate: 82.4\%). The Tanh function also exhibited a strong negative correlation with momentum (correlation coefficient: -0.79 ), and the number of iterations required to achieve a $90 \%$ training rate decreased considerably from 151 to 98 (improvement rate: 35.1\%). By contrast, the ReLU, Leaky ReLU, and ELU functions exhibited little correlation with momentum; thus, momentum changes did not 
affect the learning efficiency.

(5) This case study demonstrated the effectiveness of hyperparameter settings in MLP modeling. For example, under the proper hyperparameter settings (in this case, a learning rate of 0.1 and momentum of 0.9 ), the Sigmoid function performs effectively in terms of training accuracy and efficiency.

(6) Results from the two sets of hyperparameter settings, the initial set based on experience and the optimized set based on the recommendations in Section 4.1 to 4.3, demonstrated a testing accuracy of $87.4 \%$ and $97.2 \%$, respectively. In regards to the training efficiency performance, the number of iterations required for the initial and optimized settings to achieve $80 \%$ training accuracy was 5680 and 210 , respectively. Therefore, optimization of the hyperparameter settings improved the accuracy of model testing and reduced training time.

Acknowledgements This research was supported in part by the Frontier Mould and Die Research and Development Center from The Featured Areas Research Center Program within the framework of the Higher Education Sprout Project by the Ministry of Education (MOE), Taiwan.

Author contribution $\quad$ K.-C. Ke and M.-S. Huang were responsible for deriving formulas. K.-C. Ke was responsible for simulation. K.-C. Ke and M.-S. Huang were involved in the discussion and significantly contributed to making the final draft of the article. All the authors read and approved the final manuscript.

Funding Not applicable.

Data availability The authors confirm that the data supporting the findings of this study are available within the article.

\section{Declarations}

Ethical approval Not applicable. 
Consent to participate Not applicable.

Consent to publish Not applicable.

Competing interests The authors declare no competing interests. 


\section{Reference}

[1] Huang MS, Ke KC, Liu CY (2021) Cavity pressure-based holding pressure adjustment for enhancing the consistency of injection molding quality. J Appl Polym Sci 138:50357(110)

[2] Zhang J, Zhao P, Zhao Y, Huang J, Xia N, Fu J (2019) On-line measurement of cavity pressure during injection molding via ultrasonic investigation of tie bar. Sens Actuators Phys 285:118-126

[3] Lin CC, Wang WT, Kuo CC, Wu CL (2014) Experimental and theoretical study of melt viscosity in injection process. Int J Mech Mecha Eng 8:1-5

[4] Wang J, Peng J, Yang W (2011) Filling-to-packing switchover mode based on cavity temperature for injection molding. Polym-Plast Technol Eng 50:1273-1280

[5] Zhao P, Xia N, Zhang J, Xie J, Zhang C, Fu, J (2020) Measurement of molecular orientation using longitudinal ultrasound and its first application in in-situ characterization. Polymer 187:122092(1-11)

[6] Chang YH, Wei TH, Chen SC, Lou YF (2020) The investigation on PVT control method establishment for scientific injection molding parameter setting and its quality control. Polym Eng Sci 60:2895-2907

[7] Wang J (2012) PVT properties of polymers for injection molding. Some Critical Issues for Injection Molding 1-30

[8] Hopmann C, Kahve C, Schmitz M (2020) Development of a novel control strategy for a highly segmented injection mold tempering for inline part warpage control. Polym Eng Sci 60:2428-2438

[9] Wang J, Mao Q (2013) A novel process control methodology based on the PVT behavior of polymer for injection molding. Adv Polym Technol 32:E474-E485 
[10] Kamaruddin S, Khan ZA, Foong SH (2010) Application of Taguchi method in the optimization of injection moulding parameters for manufacturing products from plastic blend. Int J Eng Technol 2:574-580

[11] Yizong T, Ariff ZM, Khalil AM (2017) Influence of processing parameters on injection molded polystyrene using Taguchi method as design of experiment. Procedia Eng $184: 350-359$

[12] Kiatcharoenpol T, Vichiraprasert T (2018) Optimizing and modeling for plastic injection molding process using Taguchi method. J Phys Conf Ser 1026:012018

[13] Wang Q, Yang C, Du K, Wu Z (2019) Effect of micro injection molding parameters on cavity pressure and temperature assisted by Taguchi method. Mechanika 25:261-268

[14] Feng Q, Liu L, Zhou X (2020) Automated multi-objective optimization for thin-walled plastic products using Taguchi, ANOVA, and hybrid ANN-MOGA. Int J Adv Manuf Technol 106:559-575

[15] Lockner Y, Hopmann C (2021) Induced network-based transfer learning in injection molding for process modelling and optimization with artificial neural networks. Int J Adv Manuf Technol 112:3501-3513

[16] Ke KC, Huang MS (2020) Quality prediction for injection molding by using a multilayer perceptron neural network. Polymers 12:1812

[17] Ke KC, Huang MS (2021) Quality classification of injection-molded components by using quality indices, grading, and machine learning. Polymers 13:353

[18] Hwang S, Kim J (2019) Injection mold design of reverse engineering using injection molding analysis and machine learning. J Mech Sci Technol 33:3803-3812

[19] Ogorodnyk O, Lyngstad OV, Larsen M, Wang K, Martinsen K (2019) Application of machine learning methods for prediction of parts quality in thermoplastics injection molding. Advanced Manufacturing and Automation VIII. Springer, Singapore, pp 237244 
[20] Lei Y, Tang K (2021) Learning rates for stochastic gradient descent with nonconvex objectives. IEEE Trans Pattern Analysis Machine Intelligence. https://doi.org/10.1109/TPAMI.2021.3068154

[21] Cheridito P, Jentzen A, Rossmannek F (2021) Non-convergence of stochastic gradient descent in the training of deep neural networks. J Complex 64: 101540

[22] Jin R, He X (2020) Convergence of momentum-based stochastic gradient descent. 16th IEEE Int Conf Control Automation, Sapporo, Hokkaido, Japan, pp 779-784

[23] Sharma A (2018) Guided stochastic gradient descent algorithm for inconsistent datasets. Appl Soft Comput J 73:1068-1080

[24] Gupta P, Garg S (2019) Breast cancer prediction using varying parameters of machine learning models. 3rd Int Conf Computing Network Communications, Trivandrum, Kerala, India, pp 593-601

[25] Bock S, Weis M (2019) A proof of local convergence for the Adam optimizer. Int Joint Conf Neural Networks, Institute of Electrical and Electronics Engineers Inc., Budapest, Hungary

[26] Hochreiter S, (1998) The vanishing gradient problem during learning recurrent neural nets and problem solutions," Int J Uncertainty, Fuzziness Knowledge-based Systems, 6:107116

[27] Bjorck J, Gomes C, Selman B, Weinberger KQ (2018) Understanding batch normalization. ArXiv180602375 Cs Stat 